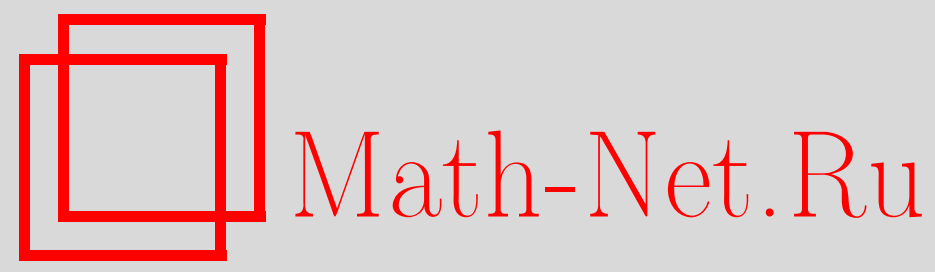

О. В. Денисов, Об алгоритме поиска существенных аргументов случайных булевых функций, Матем. вопр. криптогр., 2015, том 6, выпуск 3, 19-32

DOI: https://doi.org/10.4213/mvk158

Использование Общероссийского математического портала Math-Net.Ru подразумевает, что вы прочитали и согласны с пользовательским соглашением

http://www.mathnet.ru/rus/agreement

Параметры загрузки:

IP : 3.93 .64 .190

26 апреля 2023 г., $15: 27: 38$

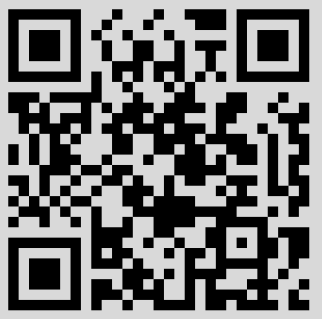


УДК: $519.719 .2+519.233 .2$

\title{
Об алгоритме поиска существенных аргументов случайных булевых функций
}

\author{
О. В. Денисов \\ ООО «Центр сертификационных исследований», Москва
}

Получено 30.V.2014

Ранее автором был предложен алгоритм поиска существенных аргументов двоичных вектор-функций по $N$ случайным векторам аргументов, имеющих равномерное распределение, и искаженным значениям функций. В настоящей статье изучается вероятность выявления первым этапом этого алгоритма всех существенных аргументов булевой функции или их части в зависимости от $N$, если функция выбирается случайно из множества симметрических либо пороговых функций. Приводится обзор нескольких работ по этой теме.

Ключевые слова: поиск существенных аргументов, случайная булева функция, оценки спектра функции

On the algorithm of detecting the essential arguments of random Boolean function

\section{O. V. Denisov}

\section{LLC «Sertification Research Center», Moscow}

\begin{abstract}
Earlier author had presented the algorithm for detection of essential arguments of the binary vector-function given $N$ random uniformly distributed arguments and distorted values of the function. The dependence on $N$ of the probability that all (or almost all) essential arguments of Boolean function $f$ are detected on the first stage of the algorithm is studied. Cases of random symmetric and random threshold functions $f$ are considered. A review of some relevant papers is given.
\end{abstract}

Key words: essential arguments detection, random Boolean function, function spectrum estimation

Citation: Mathematical Aspects of Cryptography, 2015, vol. 6, no. 3, pp. 19-32 (Russian). 


\section{Введение}

Изложим постановку задачи, рассматривавшейся в [4], ограничив ее случаем булевой функции. В ряде задач криптографии и теории информации возникает схема, представленная на рис. 1, где для наблюдения доступны вход $\vec{x}(t)$ и выход $y(t)$ схемы, $1 \leqslant t \leqslant N$. Требуется определить множество $\Theta=\left\{\theta_{1}, \ldots, \theta_{n}\right\}$ номеров существенных входов схемы.

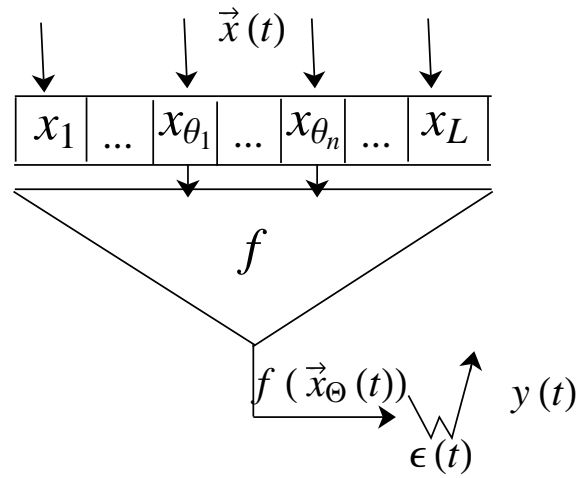

Рис. 1. Схема двоичного преобразования с искажением

Эта схема реализует булеву функцию, зависящую формально от $L$ переменных, из которых не более $n$ являются существенными. Поэтому поставленная задача может рассматриваться как задача поиска существенных аргументов булевой функции.

Пусть $\left(\mathbb{Z}_{2}, \oplus\right)$ - группа вычетов по модулю $2, \mathbb{Z}_{2}^{L}-$ множество двоичных векторов размерности $L\left(L-я\right.$ декартова степень множества $\left.\mathbb{Z}_{2}=\{0,1\}\right)$, $U\left(\mathbb{Z}_{2}^{L}\right)$ - равномерное распределение на этом множестве. Входные векторы схемы (аргументы) являются случайными, независимыми и имеющими равномерное распределение:

$$
\vec{x}(t)=\left(x_{1}(t), \ldots, x_{L}(t)\right) \sim U\left(\mathbb{Z}_{2}^{L}\right) .
$$

Булева функция $f: \mathbb{Z}_{2}^{n} \rightarrow \mathbb{Z}_{2}$ неизвестна, но известно число ее аргументов $n=$ $=|\Theta|<L$. Случайные двоичные искажения $\varepsilon(1), \ldots, \varepsilon(N)$ одинаково распределены, независимы и не зависят от всех векторов аргументов. Преобладание нуля в их распределении обозначаем через

$$
\delta:=\mathbf{E}(-1)^{\varepsilon(t)} \in[-1,1], \quad \delta \neq 0, t=1, \ldots, N .
$$

Искажения аддитивно накладываются на значения функции, и на выходе наблюдаются случайные величины

$$
y(t)=f\left(\vec{x}_{\Theta}(t)\right) \oplus \varepsilon(t), \quad 1 \leqslant t \leqslant N .
$$


Здесь и далее для произвольного вектора $\vec{x}=\left(x_{1}, \ldots, x_{M}\right)$ и непустых $I=$ $=\left\{i_{1}, \ldots, i_{r}\right\} \subset\{1, \ldots, M\}$ обозначаем через

$$
\vec{x}_{I}=\left(x_{i_{1}}, \ldots, x_{i_{r}}\right)
$$

подвектор вектора $\vec{x}$, состоящий из координат с номерами из $I$.

Условия, перечисленные в последнем абзаце, далее называем условиями (Ф) и всюду считаем их выполненными. Они задают статистическую параметрическую модель, в которой неизвестны параметры $\Theta, f$ и ненулевое значение $\delta$.

Опишем первый этап работы алгоритма, предложенного в [4]. Обозначим через

$$
\overrightarrow{x f}=\left(x_{1}, \ldots, x_{n}, f(\vec{x})\right)
$$

случайный вектор размерности $n+1$, составленный из аргументов и значения функции, при равномерном распределении аргумента $\vec{x} \sim U\left(\mathbb{Z}_{2}^{n}\right)$. Рассмотрим значения характеристической функции [1] распределения вектора $\overrightarrow{x f}$ на двоичных векторах с единственной ненулевой координатой:

$$
\varphi_{\overrightarrow{x f}}\{k\}=\mathbf{E}(-1)^{x_{k}+f(\vec{x})}=2^{-n} \sum_{\vec{a} \in \mathbb{Z}_{2}^{n}}(-1)^{a_{k}+f(\vec{a})}, \quad 1 \leqslant k \leqslant n .
$$

Эти величины являются также коэффициентами в разложении Фурье псевдобулевой функции $(-1)^{f(\vec{x})}$, т. е. частью спектра функции $f$.

Спектральные статистики из [4], принимающие здесь вид

$$
S_{N}(i):=\sum_{1 \leqslant t \leqslant N}(-1)^{x_{i}(t)+y(t)}, \quad 1 \leqslant i \leqslant L,
$$

являются суммами одинаково распределенных независимых случайных величин. При $i \notin \Theta$ среднее значение $\mathbf{E S}_{N}(i)$ равно 0 (а дисперсия равна $N$ ). Поэтому на первом этапе принимается решение о включении $i$-го входа в статистическую оценку $\Theta^{*}(1)$ для $\Theta$, если абсолютная величина статистики не меньше некоторой границы:

$$
\Theta^{*}(1)=\left\{i \in\{1, \ldots, L\}:\left|S_{N}(i)\right| \geqslant B_{1} \sqrt{N}\right\} .
$$

Далее везде полагаем для любого заданного $\alpha \in(0,1)$

$$
B_{1}=B_{1}(n, L, \alpha, N):=\sqrt{(2+\gamma) \ln \frac{4(L-n)}{\alpha}}
$$


где $\gamma^{2}:=\frac{1}{N} \max _{0 \leqslant k \leqslant n} \ln \left\{\left(\begin{array}{l}n \\ k\end{array}\right) 2^{k+2} \frac{L-n}{\alpha}\right\}$. Из [4, теорема 1] следует, что если $\gamma \leqslant 1 / 4$, то при таком выборе границы $\Theta^{*}(1)$ не содержит номеров несущественных аргументов с вероятностью не меньше $1-\alpha$ для любого заданного $0<\alpha<1$.

С другой стороны, для любого $\theta_{k} \in \Theta$

$$
\mathbf{E S}_{N}\left(\theta_{k}\right)=N \delta \varphi_{\overrightarrow{x f}}\{k\}, \quad \mathbf{D} S_{N}\left(\theta_{k}\right)=N\left(1-\delta^{2} \varphi_{\overrightarrow{x f}}^{2}\{k\}\right) .
$$

Поэтому чем больше значение $\left|\delta \varphi_{\overrightarrow{x f}}\{k\}\right|$, тем меньше материала требуется для того, чтобы $i$-й вход попал в $\Theta^{*}(1)$ с вероятностью, не меньшей заданной величины.

При дальнейших расчетах будем рассматривать схему серий, в которой вероятностная схема случайного выбора функции $f$ и параметры $\delta, L, \alpha, N$ зависят от $n$ и $n \rightarrow \infty$. При этом предполагаем выполненными условия

$$
\alpha \rightarrow 0, \frac{N}{n} \rightarrow \infty, \frac{N}{\ln \frac{L-n}{\alpha}} \rightarrow \infty .
$$

Из двух последних условий в (3) и [4, формула (10)] следует, что $\gamma \rightarrow 0$, и поэтому $\gamma \leqslant 1 / 4$,

$$
\mathbf{P}\left\{\Theta^{*}(1) \subset \Theta\right\} \geqslant 1-\alpha
$$

для всех достаточно больших $n$. При $\gamma \rightarrow 0$ имеем также

$$
B_{1}^{2} \sim 2 \ln \frac{4(L-n)}{\alpha}
$$

\section{1. Работа алгоритма при случайном выборе симметрической функции}

В [5] рассматривалась $p$-модель случайного выбора симметрической булевой функции $f$, при которой ее значения $f_{0}, \ldots, f_{n}$ на векторах веса $0, \ldots, n$ выбираются независимо по схеме Бернулли с фиксированной вероятностью единицы $p \in(0,1)$. В частности, $\frac{1}{2}$-модель задает равномерное распределение на множестве всех симметрических булевых функций от $n$ переменных.

Теорема 1. Пусть при условиях $\boldsymbol{\Phi}$ и (3) симметрическая функция $f$ выбирается случайно по р-модели. Тогда: 
1. Если $B_{1}>\sqrt{b \ln n}$ для некоторой константы $b>2 u$

$$
N / N_{\mathrm{sym}}^{(1)} \rightarrow 0, \text { где } N_{\mathrm{sym}}^{(1)}:=\frac{\left(B_{1}-\sqrt{b \ln n}\right)^{2} \sqrt{\pi n^{3}}}{\delta^{2} 6 p(1-p)},
$$

$m o \mathbf{P}\left\{\Theta^{*}(1)=\varnothing\right\} \rightarrow 1$.

2. Если для некоторой константы $b>2$

$$
N / N_{\mathrm{sym}}^{(2)} \rightarrow \infty, \quad \text { где } N_{\mathrm{sym}}^{(2)}:=\frac{\left(B_{1}+\sqrt{b \ln n}\right)^{2} \sqrt{\pi n^{3}}}{\delta^{2} 6 p(1-p)},
$$

$m o \mathbf{P}\left\{\Theta^{*}(1)=\Theta\right\} \rightarrow 1$.

Доказательство. Из теоремы 1 [5] следует, что распределение случайной величины $w_{1}(f):=\sum_{\vec{x} \in \mathbb{Z}_{2}^{n}} f(\vec{x})(-1)^{x_{1}}$ при $n \rightarrow \infty$ асимптотически нормально:

$$
\frac{w_{1}(f)}{\sqrt{p(1-p) \sigma_{1}^{2}(n)}} \stackrel{D}{\rightarrow} N(0,1)
$$

где $\sigma_{1}^{2}(n) \sim 2^{2 n} \frac{3}{2 \sqrt{\pi n^{3}}}$ согласно формуле (25) [5].

Так как $f(\vec{x})=\frac{1-(-1)^{f(\vec{x})}}{2}$, то $w_{1}(f)=-2^{n-1} \varphi$ (для краткости используем обозначение $\left.\varphi=\varphi_{\overrightarrow{x f}}\{1\}\right)$. Тогда

$$
\frac{w_{1}^{2}(f)}{p(1-p) \sigma_{1}^{2}(n)} \sim \frac{2^{2 n} \varphi^{2} 2 \sqrt{\pi n^{3}}}{4 p(1-p) 2^{2 n} 3}=\frac{\varphi^{2}}{a(n)},
$$

где $a(n):=6 p(1-p) / \sqrt{\pi n^{3}}$. Отсюда с учетом теоремы непрерывности [2, теорема 4a, c. 124] следует, что распределение последовательности случайных величин $\varphi^{2} / a(n)$ сходится к распределению хи-квадрат с одной степенью свободы. Тогда для любой числовой последовательности $0<\varkappa(n) \rightarrow+\infty$ выполняется соотношение

$$
\mathbf{P}\left\{\frac{1}{\varkappa(n)} \leqslant \frac{\varphi^{2}}{a(n)} \leqslant \varkappa(n)\right\} \rightarrow 1 .
$$


1. Докажем первое утверждение теоремы. Пусть $\varkappa(n):=N_{\mathrm{sym}}^{(1)} / N \rightarrow \infty$. Очевидно, что

$$
\begin{aligned}
\mathbf{P}\left\{\Theta^{*}=\varnothing\right\}=\mathbf{P}\left\{\Theta^{*}\right. & \left.=\varnothing, \frac{\varphi^{2}}{a(n)} \leqslant \varkappa(n)\right\}+\mathbf{P}\left\{\Theta^{*}=\varnothing, \frac{\varphi^{2}}{a(n)}>\varkappa(n)\right\} \geqslant \\
& \geqslant \mathbf{P}\left\{\frac{\varphi^{2}}{a(n)} \leqslant \varkappa(n)\right\} \mathbf{P}\left\{\Theta^{*}=\varnothing \mid \frac{\varphi^{2}}{a(n)} \leqslant \varkappa(n)\right\} .
\end{aligned}
$$

Согласно (6) в последнем произведении первая вероятность стремится к 1, и достаточно доказать, что вторая вероятность тоже стремится к 1. Для этого воспользуемся теоремой 2 [4]. Ее условия ВФ и (13) соответствуют нашим условиям $\boldsymbol{\Phi}$ и (3). Проверим, что $N$ удовлетворяет условиям п. 1 этой теоремы: $N \leqslant N_{1}=\frac{\left(B_{1}-\sqrt{b \ln n}\right)^{2}}{\Psi_{\max }^{2}}$, где

$$
\Psi_{\max }^{2}=\max _{1 \leqslant k \leqslant n} \varphi_{\overrightarrow{x f}}^{2}\{k\} \delta^{2}=\varphi^{2} \delta^{2}
$$

с учетом первого равенства в (2) и симметричности функции $f$.

Действительно, в силу неравенства $\frac{\varphi^{2}}{a(n)} \leqslant \varkappa(n)$

$$
N=\frac{N_{\mathrm{sym}}^{(1)}}{\varkappa(n)}=\frac{\left(B_{1}-\sqrt{b \ln n}\right)^{2}}{\delta^{2} a(n)} \frac{1}{\varkappa(n)} \leqslant \frac{\left(B_{1}-\sqrt{b \ln n}\right)^{2}}{\delta^{2} a(n)} \frac{a(n)}{\varphi^{2}}=N_{1} .
$$

Тогда $\mathbf{P}\left\{\Theta^{*}(1)=\varnothing\right\} \rightarrow 1$ согласно п. 1 теоремы 2 [4]. Первое утверждение теоремы доказано.

2. Аналогично докажем второе утверждение теоремы. Пусть $\varkappa(n)=$ $=N / N_{\mathrm{sym}}^{(2)} \rightarrow \infty$. Тогда

$$
\mathbf{P}\left\{\Theta^{*}=\Theta\right\} \geqslant \mathbf{P}\left\{\frac{\varphi^{2}}{a(n)} \geqslant \frac{1}{\varkappa(n)}\right\} \mathbf{P}\left\{\Theta^{*}=\Theta \mid \frac{\varphi^{2}}{a(n)} \geqslant \frac{1}{\varkappa(n)}\right\} .
$$

Здесь также с учетом (6) достаточно доказать, что последняя вероятность стремится к 1. Для этого проверяем, что $N$ удовлетворяет условиям п. 2 теоремы 2 [4]:

$$
N \geqslant N_{2}=\frac{\left(B_{1}+\sqrt{b \ln n}\right)^{2}}{\Psi_{\min }^{2}}, \text { где } \Psi_{\min }^{2}=\min _{1 \leqslant k \leqslant n} \varphi_{\vec{x}}^{2}\{k\} \delta^{2}=\varphi^{2} \delta^{2} .
$$


Оно выполнено, поскольку

$$
N=N_{\mathrm{sym}}^{(2)} \varkappa(n) \geqslant \frac{\left(B_{1}+\sqrt{b \ln n}\right)^{2}}{\delta^{2} a(n)} \frac{a(n)}{\varphi^{2}}=N_{2} .
$$

Тогда $\mathbf{P}\left\{\Theta^{*}(1)=\Theta\right\} \rightarrow 1$ согласно п. 2 теоремы 2 [4]. Теорема 1 доказана.

Определим пороговое значение $N$, достаточное для успешной работы алгоритма, по аналогии с классическим в вероятностной комбинаторике подходом (см. [10]). Функцию $N_{\text {rou }}(n)$ назовем грубым (rough) порогом объема материала для события $A=\left\{\Theta^{*}=\Theta\right\}$, если

$$
\lim _{n \rightarrow \infty} \mathbf{P}(A)=\left\{\begin{array}{l}
1, \text { если } N(n) / N_{\text {rou }}(n) \rightarrow \infty, \\
0, \text { если } N(n) / N_{\text {rou }}(n) \rightarrow 0 .
\end{array}\right.
$$

Функция $N_{\text {rou }}(n)$ определена не однозначно; в частности, любая функция, полученная из нее умножением на функцию, стремящуюся к ненулевой константе, также является грубым порогом.

При выводе формулы для грубого порога используется следующее условие [4, (14)]:

$$
\frac{\ln \frac{L-n}{\alpha}}{\ln n} \rightarrow \infty
$$

Оно означает, что асимптотически величины $N_{\text {sym }}^{(1)}$ и $N_{\text {sym }}^{(2)}$ эквивалентны.

Следствие 1. Если при условиях теоремы 1 дополнительно выполнено условие (8), то функция

$$
N_{\text {sym }}(n):=\frac{B_{1}^{2} \sqrt{\pi n^{3}}}{\delta^{2} 6 p(1-p)} \sim \frac{\sqrt{\pi n^{3}}}{3 \delta^{2} p(1-p)} \ln \frac{4(L-n)}{\alpha}
$$

является грубым порогом объема материала в смысле определения (7).

Доказательство. Утверждение следует из теоремы 1 и того, что $N_{\text {sym }}^{(1)}$ и $N_{\text {sym }}^{(2)}$ эквивалентны величине $N_{\text {sym }}(n)$. Асимптотическая формула для $N_{\text {sym }}(n)$ следует из (5).

Замечания. 1. Полученная функция $N_{\text {sym }}(n)$ на величину порядка $n^{1 / 2}$ больше порога $N_{\text {maj }}$ (см. ниже формулу (12)), рассчитанного в [4] для случая, когда $f$ - мажоритарная функция, т. е. симметрическая пороговая функция, преобладание распределения значений которой близко к нулю.

2015, T. 6, № 3, C. 19-32 
2. В [4] пороги объема материала рассчитывались для случая булевых функций $f$ с одинаковыми и неслучайными значениями модулей спектральных коэффициентов $\varphi_{\overrightarrow{x f}}\{i\}, 1 \leqslant i \leqslant n$. Так как вероятности событий $\left\{\left|S_{N}(i)\right| \geqslant B_{1} \sqrt{N}\right\}$ зависят лишь от этих параметров функции $f$, то распределение случайного множества $\Theta^{*}(1)$ и вероятность события $A$, т. е. успешного окончания алгоритма на первом этапе, одинаковы для всех таких функций.

3. Если функция выбирается из класса функций, у которых наборы модулей таких спектральных коэффициентов различны, то вероятности $\mathbf{P}(A)$ разные для разных функций. Чтобы по-прежнему характеризовать алгоритм порогом объема материала, можно выбирать $f$ из класса случайно. В этом случае вероятностная схема становится двухэтапной и строится на прямом произведении вероятностных пространств: сначала выбирается $f$, а затем независимо от нее - входные векторы и искажения. Тогда распределение $\Theta^{*}(1)$ оказывается вероятностной смесью распределений, соответствующих всем функциям из класса. Это усложняет поиск порога объема в смысле [4], и здесь мы ограничились расчетом грубого порога объема (7).

4. Отметим, что вероятность успешной работы алгоритма связана не только со случайным выбором векторов аргументов, но и со случайным выбором функции $f$. Поэтому при условиях п. 2 теоремы 1 существуют симметрические функции, для которых результатом алгоритма является $\Theta^{*}(1) \neq \Theta$, но суммарная мера таких функций стремится к нулю.

\section{2. Работа алгоритма при случайном равновероятном выборе пороговой функции}

Напомним, что булева функция $f$ называется пороговой (threshold), если для нее справедливо следующее действительнозначное представление: для некоторых $a_{1}, \ldots, a_{n}, b \in \mathbb{R}$

$$
f\left(x_{1}, \ldots, x_{n}\right)=\mathbb{I}\left\{a_{1} x_{1}+\ldots+a_{n} x_{n} \geqslant b\right\}, \quad x_{1}, \ldots, x_{n} \in\{0,1\} .
$$

Получим нижнюю оценку для числа существенных входов, которые будут найдены на первом этапе алгоритма, при случайной пороговой функции $f$. Чтобы упростить запись формул, будем использовать обозначение $\exp _{2} x:=$ $=2^{x}$.

Теорема 2. Пусть при условиях Ф функиия $f$ выббрается случайно равновероятно из множества всех пороговых функций от $n$ переменных,

$$
\alpha \rightarrow 0, \quad \frac{\ln \frac{L-n}{\alpha}}{N} \rightarrow 0 \text { при } n \rightarrow \infty
$$


и для некоторой константы $b>2$ при достаточно больших $n$ выполнено условие

$$
N \geqslant N_{\text {thr }}(n):=\frac{\left(B_{1}+\sqrt{b \ln n}\right)^{2}}{4 \delta^{2}} \exp _{2}\left(\sqrt{32 n\left(\log _{2} n+\ln \ln n\right)}\right) .
$$

Тогда $\mathbf{P}\left\{\Theta^{*}(1) \subset \Theta,\left|\Theta^{*}(1)\right| \geqslant n-\sqrt{2 n\left(\log _{2} n+\ln \ln n\right)}\right\} \rightarrow 1$.

Если при этом

$$
\log _{2} \ln \frac{L-n}{\alpha}+\log _{2}|\delta|=o\left(\sqrt{n \log _{2} n}\right)
$$

mo $\log _{2} N_{\text {thr }}(n) \sim \sqrt{32 n \log _{2} n}$.

Доказательство. 1. Формула (18) статьи [9] означает, что при $n \rightarrow \infty$ для произвольной последовательности $0<\varepsilon(n)<1, \varepsilon(n) \log _{2} n \rightarrow \infty$, почти все пороговые функции от $n$ переменных имеют не менее

$$
r(n):=n-2 \sqrt{n(2+\varepsilon(n)) \log _{2} n}
$$

коэффициентов Чоу

$$
\Delta_{i}:=\sum_{\vec{x}: f(\vec{x})=1}(-1)^{x_{i}+1}=\sum_{\vec{x} \in \mathbb{Z}_{2}^{n}}(-1)^{x_{i}+1} \frac{1}{2}\left(1+(-1)^{f(\vec{x})+1}\right)=2^{n-1} \varphi_{\overrightarrow{x f}}\{i\},
$$

абсолютная величина которых не меньше $2^{r(n)}, 1 \leqslant i \leqslant n$.

Положим $\varkappa(n):=\ln \ln n$, тогда последовательность $\varepsilon(n):=\frac{2 \varkappa(n)}{\log _{2} n}$ удовлетворяет вышеприведенным условиям. Пусть также

$$
\begin{gathered}
\varphi(n):=2 \exp _{2}\left(-2 \sqrt{2 n\left(\log _{2} n+\varkappa(n)\right)}\right), \\
I(f, \varphi):=\left\{i \in\{1, \ldots, n\}:\left|\varphi_{\overrightarrow{x f}}\{i\}\right| \geqslant \varphi(n)\right\}= \\
=\left\{i:\left|\Delta_{i}\right| \geqslant \exp _{2}\left(n-2 \sqrt{2 n\left(\log _{2} n+\varkappa(n)\right)}\right)\right\},
\end{gathered}
$$

$F(n, \varphi)-$ множество пороговых функций от $n$ переменных, для которых $|I(f, \varphi)| \geqslant r(n)$.

Из равенства $r(n)=n-2 \sqrt{2 n\left(\log _{2} n+\varkappa(n)\right)}$ и первого абзаца доказательства следует, что $\mathbf{P}\{f \in F(n, \varphi)\} \rightarrow 1$. 
2. Обозначая для $f \in F(n, \varphi)$

$$
\psi_{\min }(f):=\min _{i \in I(f)}\left|\varphi_{\overrightarrow{x f}}\{i\} \delta\right| \geqslant \varphi(n)|\delta|,
$$

находим, что при $N \geqslant N_{\mathrm{thr}}=\frac{\left(B_{1}+\sqrt{b \ln n}\right)^{2}}{\varphi(n)^{2} \delta^{2}}$ справедлива оценка

$$
N \geqslant \frac{\left(B_{1}+\sqrt{b \ln n}\right)^{2}}{\psi_{\text {min }}^{2}(f)} .
$$

Заметим, что выполняются все три условия из (3): первое и третье условия приведены в (9), а второе следует из неравенства $N \geqslant N_{\mathrm{thr}}(n)$. Тогда аналогично п. 4 доказательства теоремы 2 [4] получаем, что асимптотическое соотношение $\mathbf{P}\left\{I(f, \varphi) \subset \Theta^{*}(1)\right\} \rightarrow 1$ выполнено равномерно по $f \in F(n, \varphi)$. Итак,

$$
\mathbf{P}\left\{\left|\Theta^{*}(1)\right| \geqslant r(n)\right\} \geqslant \mathbf{P}\{f \in F(n, \varphi)\} \mathbf{P}\left\{I(f, \varphi) \subset \Theta^{*}(1) \mid f \in F(n, \varphi)\right\} \rightarrow 1 .
$$

Осталось заметить, что $\mathbf{P}\left\{\Theta^{*}(1) \subset \Theta\right\} \geqslant 1-\alpha(n) \rightarrow 1$ в силу (4). Первое утверждение теоремы доказано.

3. Докажем второе утверждение теоремы. С учетом (5) имеем

$$
\begin{aligned}
& \log _{2} N_{\mathrm{thr}}(n)= \\
&=\log _{2} B_{1}^{2}+2 \log _{2}\left(1+\sqrt{\frac{b \ln n}{B_{1}^{2}}}\right)-2 \log _{2} \delta+\sqrt{32 n \log _{2} n(1+o(1))}= \\
& \quad=\log _{2} \ln \frac{L-n}{\alpha}+O(1)+2 \log _{2} \delta+\sqrt{32 n \log _{2} n(1+o(1))}
\end{aligned}
$$

Отсюда и из (10) вытекает требуемое асимптотическое соотношение.

Теорема 2 доказана.

Из теоремы можно сделать следующий качественный вывод. Если выполнено (9), то при $N \geqslant N_{\text {thr }}(n), b>2$, для почти всех пороговых функций от $n$ переменных почти всегда $\Theta^{*}(1)$ содержит почти все существенные входы схемы и не содержит несущественные. 


\section{3. Замечания}

Перечислим некоторые работы, близкие по направлению или методам исследования к [4] и к этой статье.

В криптографических приложениях схема, изображенная на рис. 1, может рассматриваться как модель комбинирующего генератора, в которой неизвестно, выходы каких регистров сдвига используются при выработке искаженной выходной псевдослучайной последовательности. Использованные при построении алгоритма статистики $S_{N}(i)$ близки к корреляционным статистикам Зигенталера [11]. В отечественной криптографии статистические методы оценки $\Theta$ в схеме, полученной из схемы рис. 1 заменой «параллельного» входа на последовательный (вероятностная модель фильтрующего генератора), разрабатывались в статьях И. Н. Коваленко, Ю. П. Шалимова и других авторов, опубликованных в 1960-1970-х годах в ведомственных сборниках. Развиваемые в [4] методы изучения совместного распределения входа и выхода схемы с $m$-мерным выходом могут быть полезны при анализе блочных шифров, в которых S-блоки являются или биективными функциями, или бент-функциями.

Задачей определения существенных входов (переменных) булевой функции в связи с задачей выделения $n$ значимых факторов из большого числа $L$ всех факторов при планировании эксперимента занимались разные авторы - см. библиографию в $[6,7]$. В основном рассматривался случай активного эксперимента, когда наблюдатель выбирает значения входных векторов $\vec{x}(1), \ldots, \vec{x}(N)$. Если эта последовательность выбрана заранее, то эксперимент называется статическим, в противном случае - динамическим.

В [3] рассматриваются оба типа активного наблюдения над функцией (которая может быть неизвестна, как отмечается в [3, с.88]) с неискаженными значениями при известном $n$. Получены оценки для чисел $N_{\text {sta }}$ и $N_{\text {din }}$ наблюдений, достаточных для определения всех существенных входов. Доказывается, что при $L \rightarrow \infty$ и фиксированной $f$ выполнено

$$
N_{\text {sta }} \sim c(f) \log _{2} L
$$

где $n \leqslant c(f) \leqslant 2^{n}-$ некоторая характеристика функции $f$. Верхняя оценка для $N_{\text {sta }}$ получена неконструктивным методом «случайного выбора», т. е. без явного построения алгоритма.

Для динамического наблюдения справедливы асимптотические оценки

$$
n \log _{2} L(1+o(1)) \leqslant N_{\text {din }} \leqslant c(f)\left(\log _{2} L\right)^{r(f)}(1+o(1)),
$$


где $r(f)$ - целое, $1 \leqslant r(f) \leqslant n / 2$ в случае симметрической функции, $1 \leqslant r(f) \leqslant n-1$ в случае произвольной функции.

Проведем сравнение этих оценок с полученными в [4] порогами объема при выборе в качестве $f$ бент-функции либо мажоритарной функции:

$$
N_{\text {bent }}=\frac{2^{n+1} \ln (4(L-n) / \alpha)}{\delta^{2}}, \quad N_{\text {maj }}=\frac{\pi n}{\delta^{2}} \ln \frac{4(L-n)}{\alpha} .
$$

Порядок роста величин $N_{\text {sta }}$ и $N_{\text {din }}$ по $L$ всегда не меньше $\ln L$, а в (12) не больше $\ln L$. Кроме того, порядок роста величин (12) по $L$ может быть меньше $\ln L$, например при $\ln (L-n)=o(\ln L)$. Если в последнем случае $\ln \alpha=o(\ln L), \delta$ не стремится к нулю, то $N_{\text {maj }} / N_{\text {sta }} \rightarrow 0$. Порядок роста величин (12) по $n$ лежит в пределах от $n$ до $2^{n}$, что совпадает с оценками для коэффициента $c(f)$.

В [8] рассматривается случай искажения значений неизвестной функции при передаче их по двоичному каналу без памяти. (Отметим, что наше условие аддитивного наложения соответствует случаю симметричного канала.) Предложена трехэтапная рандомизированная процедура динамического поиска, в которой существенно используются значения переходных вероятностей канала. Доказано, что при некотором выборе параметров процедуры при $N \rightarrow \infty$ вероятность ее ошибки будет не более заданного значения $\varepsilon$, а отношение среднего числа необходимых наблюдений к $n \log _{2} L$ не превосходит $(1+\varepsilon) / C$, где $C-$ пропускная способность канала. С учетом (11) данное свойство названо асимптотической оптимальностью процедуры. В [7] результаты [8] обобщаются на случай $k$-значной функции от двоичных аргументов.

В упомянутых статьях рассматриваются в основном последовательные методы поиска, и требуемое число наблюдений является случайной величиной. При нашей постановке $N$ считается данным параметром вероятностной схемы, зависящим от номера серии. При такой постановке в ряде комбинаторно-вероятностных задач возникает явление фазового перехода: предел вероятности некоторого события меняется с 0 на 1 при переходе предельного соотношения между параметрами через некоторый порог.

В [10, с.99] даны два определения пороговой функции: пороговой функции вида (7) («грубой» в наших терминах) и регулярной пороговой функциии, определяемой как пара таких функций $\left(N_{\mathrm{th}}, M_{\mathrm{th}}\right)$, что

$$
\text { если }\left(N-N_{\text {th }}\right) / M_{\text {th }} \rightarrow x, \text { то } \mathbf{P}(A) \rightarrow F(x), \quad x \in \mathbb{R},
$$

где $F(x)$ - функция распределения некоторой невырожденной вероятностной меры на действительной прямой. 
Исследуемая в [4] пороговая функция является промежуточной между грубой и регулярной, поскольку при $M_{\mathrm{th}}=o\left(N_{\mathrm{th}}\right)$ (что, как правило, выполнено) справедлива цепочка импликаций:

$\left(N_{\text {th }}-\right.$ регулярный порог $) \Rightarrow\left(N_{\text {th }}-\right.$ порог $) \Rightarrow\left(N_{\text {th }}-\right.$ грубый порог $)$.

В заключение отметим некоторые возможные направления развития результатов. В случае выбора спектрально 1-однородных функций [4] интересен вопрос о существовании регулярной пороговой функции. При выборе случайной пороговой функции (а также случайной вектор-функции $f: \mathbb{Z}_{2}^{n} \rightarrow \mathbb{Z}_{2}^{m}$ ) интересен вопрос о существовании хотя бы грубой пороговой функции, поскольку в этом случае изучение осложняется большим набором ситуаций, возникающих в алгоритме, когда на первом этапе определяется одна часть входов, на втором - другая и т. д.

Другое направление связано с модификацией алгоритма в соответствии со следующими соображениями. Пусть булева функция $f$ является корреляционно-иммунной порядка $k$. Тогда по спектральному критерию $\varphi_{\overrightarrow{x f}}(I)=0$ для всех $1 \leqslant|I| \leqslant k$, и согласно теореме 2 [4] с вероятностью, стремящейся к 1, алгоритм не найдет ни одного существенного входа. Улучшить характеристики алгоритма можно, увеличив до $k+1$ число номеров входов, опробуемых на первом этапе. Верхняя оценка временной сложности алгоритма при этом увеличится. Вопрос состоит в том, можно ли выбрать граничные функции $B(G)[4]$ так, чтобы при той же верхней границе $\alpha$ (для вероятности получить несущественные выходы в $\left.\Theta^{*}\right)$ пороговые функции объема материала уменьшились хотя бы для рассмотренных здесь классов функций.

Автор благодарен А.М.Зубкову за ряд полезных замечаний, способствовавших улучшению статьи.

\section{Список литературы}

[1] Амбросимов А.С., “Свойства бент-функций q-значной логики над конечными полями”, Дискретная математика, 6:3 (1994), 50-60.

[2] Боровков А. А., Теория вероятностей, М.: Эдиториал УРСС, 1999, 472 с.

[3] Викторова И.И., Леонтович А.М., “О числе проверок для определения значимых переменных булевой функции”, Проблемы передачи информации, 14:4 (1978), 85-97.

[4] Денисов О.В., “Статистическая оценка множества существенных аргументов двоичной вектор-функции с искаженными значениями”, Математические вопросы криптографии, 5:4 (2014), 41-61. 
[5] Ивченко Г. И., Медведев Ю. И., Миронова В. А., “Анализ спектра симметрических булевых функций”, Математические вопросы криптографии, 4:1 (2013), 59-76.

[6] Малютов М.Б., “О рандомизированном планировании отсеивающих экспериментов”, Сб. «Планирование и автоматизация в научных исследованиях», М.: Сов. радио, 1974, 60-72.

[7] Малютов М.Б., Цитович И. И., “Последовательный поиск существенных переменных неизвестной функции”, Проблемы передачи информации, 33:4 (1997), 88-107.

[8] Цитович И. И., “Последовательный план для определения значимых переменных булевской функции”, Сб. «Анализ систем информатики», М.: Наука, 1991, $10-16$.

[9] Шабанин О.В., “О сложности дизъюнктивной нормальной формы пороговых функций”, Дискретная математика, 12:2 (2000), 85-92.

[10] Эрдеш П., Спенсер Дж., Вероятностные методы в комбинаторике, М.: Мир, 1976, $131 \mathrm{c}$.

[11] Siegenthaler T., "Descrypting a class of stream ciphers using ciphertext only", IEEE Trans. Computers, 34:1 (1985), 81-85. 